Supporting Information to:

\title{
One-step Routes from Di- and Triblock Copolymer Precursors to Hydrophilic Nanoporous Poly(acrylic acid)-b-Polystyrene
}

Estimation of the inter-lamellae spacing in the collapsed state for AS1-HF. The volume fractions of PS and PtBA in the precursor block copolymer AS-1 are 0.45 and 0.55 , respectively, as calculated from data in tables 1 and 2 of the paper. The average lamella spacing in AS-1 is $\mathrm{d}^{*}=25.0 \mathrm{~nm}$ (see table 1). The PS and PtBA average lamella thicknesses are therefore $11.3 \mathrm{~nm}$ and $13.7 \mathrm{~nm}$, respectively. After the HF acydolysis of the PtBA, $44 \%$ of the block mass is removed. In the collapsed state the density of the PAA is not known; it is expected to be between 1.0 and $1.2 \mathrm{~g} / \mathrm{cm}^{3}$ (this last being the literature density value of the high molecular weight PAA homopolymer). The lamella spacing in the collapsed state is estimated between 19.0 and $17.6 \mathrm{~nm}$, depending on the PAA density. The corresponding central q-value is $0.345 \mathrm{~nm}^{-1}$. This value and its first two multiples are marked by the filled diamonds in the figures $4 \mathrm{a}$ and $7 \mathrm{a}$ of the paper.

Stability of the AS-1-HF. AS1 film was put between two microscope slides with $0.5 \mathrm{~mm}$ thick aluminum foil as spacer. After heating under nitrogen at $140{ }^{\circ} \mathrm{C}$ for 25 minutes, AS1 film was oscillatory shear aligned by moving the two microscope slides with shear amplitude roughly $1000 \%$. SAXS 1D profiles of shear aligned AS1 sample and one AS1 sample without shearing are shown in Figure S1. For the shear aligned AS1 sample, the X-ray beam is normal to the shear planes. The missing of structural features in the scattering pattern from shear aligned sample indicates a good shear alignment. 


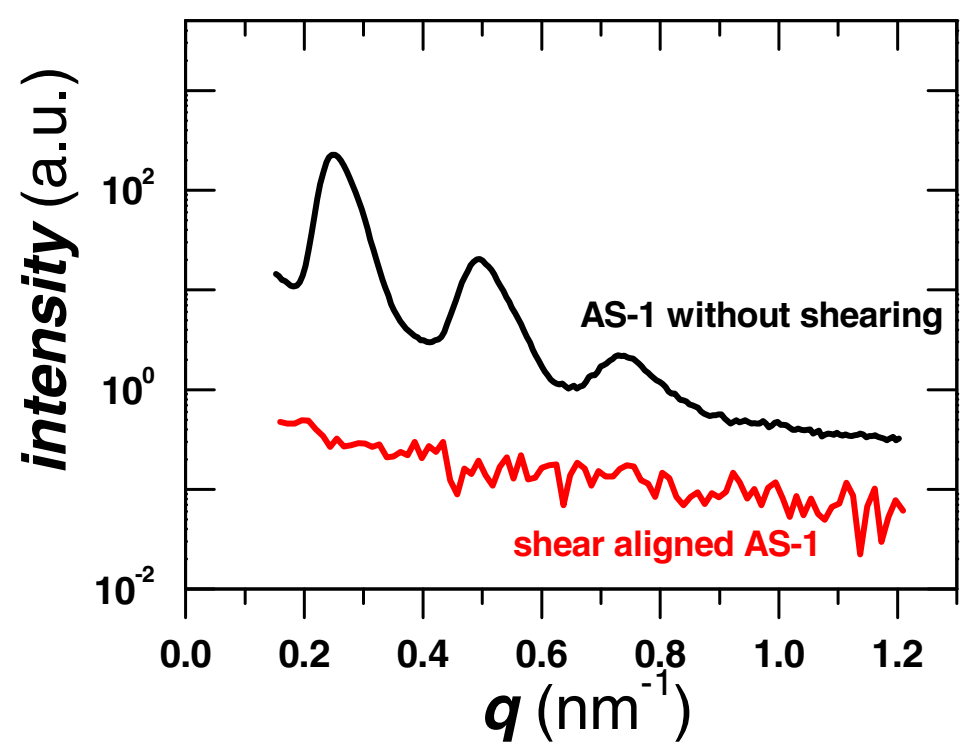

Figure S1. SAXS 1D profiles for AS-1 without shearing (top line) and shear aligned AS1 (bottom line). The X-ray beam was normal to the shear planes in the last case.

The shear aligned AS1 sample was placed in large molar excess of TFA relative to $t \mathrm{Bu}$ groups for 3 hours on a shaker. For comparison one AS1 sample without shearing was also placed in TFA for 3 hours. The pictures of shear aligned AS1 and AS1 without shearing in TFA for 30 minutes are shown in Figure S2. We can clearly see that AS1 sample without shearing at the left keeps its shape in TFA after 30 minutes; while the shear aligned AS1 at right has totally disintegrated after 30 minutes in TFA forming a hazy solution. 


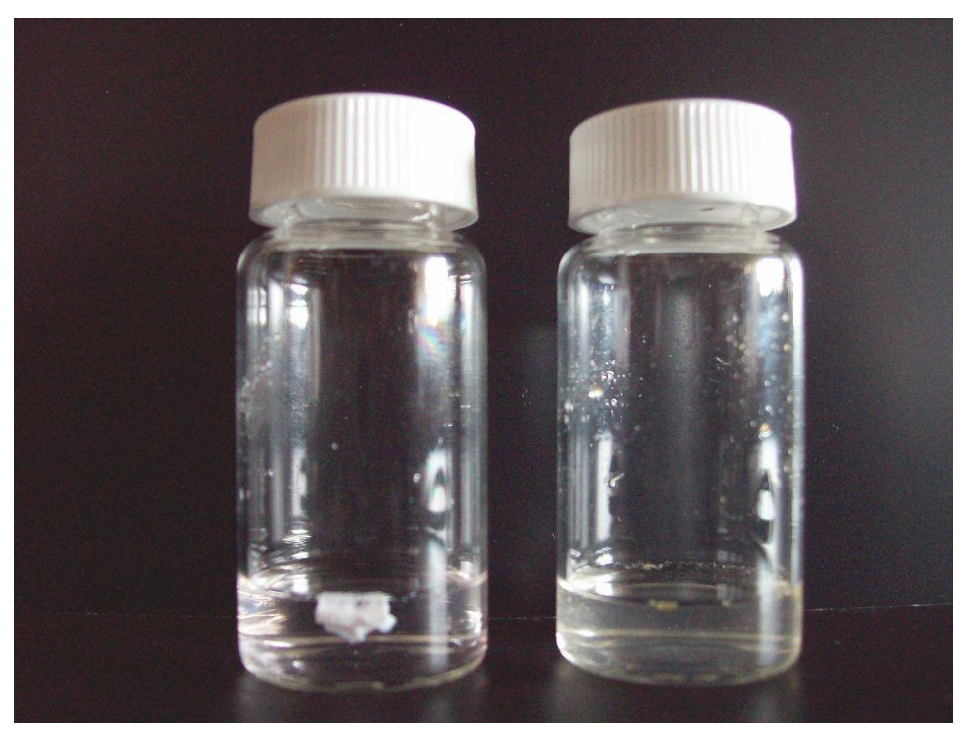

Figure S2. AS1 without shearing in TFA for 30 mins (left) and shear aligned AS1 in TFA for 30 mins (right).

FT-IR spectra of AS-1 without shearing before and after TFA treatment for 3 hours are shown in Figure S3. The characteristic peaks for PS remained intact and peaks originating from tert-butyl group in PtBA disappeared entirely. The sharp peak (a in Figure $\mathrm{S} 2)$ corresponding to the ester carbonyl group $(\mathrm{C}=\mathrm{O})$ was broadened after HFtreatment due to the formation of carboxylic acid carbonyl group. 


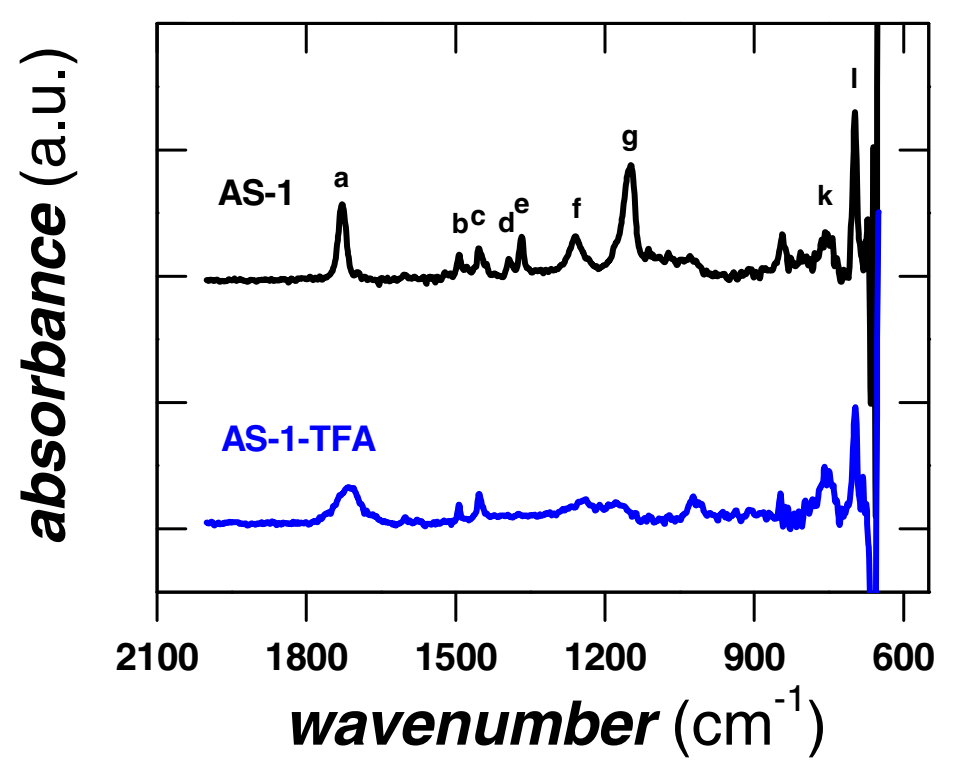

Figure S3. FT-IR spectra of AS-1 precursor sample (top line), after TFA treatment (bottom line). Peaks are identified as follows: (a) $\mathrm{C}=\mathrm{O}$ stretching $\left(1730 \mathrm{~cm}^{-1}\right)$ in ester group, (b and c) $\mathrm{CH}$ stretching $\left(1489 \mathrm{~cm}^{-1}, 1454 \mathrm{~cm}^{-1}\right)$ in monosubstituted phenyl group, (d and e) $\mathrm{CH}_{3}$ bending $\left(1394 \mathrm{~cm}^{-1}, 1368 \mathrm{~cm}^{-1}\right)$ in $t \mathrm{Bu}$ group, (f) C-C-O stretching (1259 $\left.\mathrm{cm}^{-1}\right)$ in O-tBu, (g) C-O stretching $\left(1151 \mathrm{~cm}^{-1}\right)$ in $\mathrm{O}-t \mathrm{Bu}$, and $(\mathbf{k}$ and $\mathbf{l})$ monosubstituted phenyl group $\left(760 \mathrm{~cm}^{-1}, 700 \mathrm{~cm}^{-1}\right)$. 